\title{
Left Atrial Size and Risk of Stroke in Patients with Sinus Rhythm and Normal Left Ventricular Systolic Function
}

\author{
Hanan K. Altalhi ${ }^{1,2}$, Asgad A. Abdalgbar ${ }^{1,2}$, Ali Areef Fadhlullah ${ }^{1,2}$ \\ ${ }^{1}$ Faculty of Medicine, University of Omar El mukhtar, Albayda, Libya \\ ${ }^{2}$ Department of Internal Medicine, University of Omar El mukhtar, Albayda, Libya
}

Email address:

hnangk@yahoo.com (H. K. Altalhi), aabdalgbar@yahoo.com (A. A. Abdalgbar), ali.aref67@yahoo.com (A. A. Fadhlullah)

\section{To cite this article:}

Hanan K. Altalhi, Asgad A. Abdalgbar, Ali Areef Fadhlullah. Left Atrial Size and Risk of Stroke in Patients with Sinus Rhythm and Normal Left Ventricular Systolic Function. American Journal of Internal Medicine. Vol. 4, No. 6, 2016, pp. 113-116. doi: $10.11648 /$ j.ajim.20160406.14

Received: October 21, 2016; Accepted: November 2, 2016; Published: November 25, 2016

\begin{abstract}
Increased left atrial (L A) size associated with poor cardiovascular outcome such as development of heart failure, atrial fibrillation (AF) and stroke in the elderly. Objective: The present study was conducted to reevaluate the relationship between la size and stroke in subjects of all ages, not just the elderly who presented with preserved left ventricular systolic function (lvsf) and sinus rhythm (sr). Patients and Methods: This is case control study of 52 patients admitted to the hospital 26 with diagnosis of ischemic stroke (case subject), and 26 patients without ischemic stroke were included as (control subject). The diagnosis of Cerebral infarction was confirmed by a new computer Tomography of Brain All participant underwent the standard examination and testing as well as Echocardiography (measurement of left atrial dimension, Ejection Fraction,Mitral inflow, LV mass), patients with valvular heart disease, atrial fibrillation or coronary disease were excluded. Result: The mean age was $69 \pm 18$ years in the stroke group and $66 \pm 17$ years in control group. The LA dimension of stroke group $(3.38 \pm 0.50$ $\mathrm{cm})$ was significantly greater than that of the control group $(3.56 \pm 0.44 \mathrm{~cm}) ; \mathrm{P}<0.0001)$ E\&A Velocity showed no significant relation with Stroke $(\mathrm{p}=\mathrm{NS})$ Left ventricular mass, left ventricular mass / height and left ventricular mass index were significantly related to stroke $(\mathrm{p}<0.0001, \mathrm{p}=0.001, \mathrm{p}=0.001$, respectively). Conclusion: Echocardiographic left atrial enlargement has prognostic value in identifying a subset of persons at increased risk of stroke.
\end{abstract}

Keywords: Left Atrial Size (La), Left Ventricular Systolic Function (Lvsf) Sinus Rhythm (Sr), Stroke Left Ventricular Mass Index (Lvmi), Echocardiography (Echo)

\section{Introduction}

Increased left atrial (LA) size associated with poor outcome such as development of heart failure, atrial fibrillation (AF), and stroke in the elderly, even in case in which sinus rhythm is maintained (Abhayratna WP, et al 2006). There is already strong evidence for an association between AF and ischemic stroke, and the majority of strokes occurred in patients who presented with sinus rhythm (American Heart association. 2003). LA size is regarded as reflection of the average effect of left ventricular (LV) filling pressure against the LA over time. And has been proposed as marker of diastolic burden (Douglas PS. 2003). In addition, increased LV filling pressure resulting from a decrease in myocardial contractility or impairment of myocardial relaxation is considered the main pathophysiological factor involved in heart failure and contributes to enlargement of the LA (Tsang SM, et al 2002). To minimize the impact of LV filling pressure on LA size, the present study was performed in subjects presenting with preserved left ventricular systolic function (LVSF).

\section{Objective}

The present study was conducted to reevaluate the relationship between LA size and stroke in subjects of all ages, not just the elderly who presented with preserved LVSF and sinus rhythm 


\section{Patients and Methods}

This is case control study of 26 patients admitted to the hospital with diagnosis of ischemic infarction (Case subject) and 26 patients without ischemic infarction were included as (control subjects.) Date were collected through interview cases and controls, physical and neurological examination by study physicians. The diagnosis of cerebral infarction was confirmed by new computed tomography (CT) of brain.

Subjects with LV ejection fraction $<50 \%$ and valvular heart disease or structural abnormalities or history of coronary artery disease or Q wave on ECG were excluded from the study.

Transthoracic Echocardiography was performed in all subjects LVEF was estimated using the Simpson rule. Patients with LVEF $<50 \%$ were exclude from the study (Lang RM., et al 2005)

Mitral inflow was assessed by pulsed-wave Doppler echocardiography from the apical four-chamber view, with the Doppler beam aligned parallel to the direction of flow and with a sample volume of $1-2 \mathrm{~mm}$ at the mitral tips. From the mitral inflow profile, $\mathrm{E}$ and $\mathrm{A}$ wave velocities, declaration time (DT), and the E/A ratio were measured (Lang RM, et al 2005)

LA diameter was measured using M-mode, from the posterior aortic wall to the posterior left atrial wall, in the parasternal long - axis view at end -ventricular systole, (ie; just before the mitral valve opening), left atrial enlargement was defined as left atrial diameter of $4.1 \mathrm{~cm}$ or greater in men or $3.9 \mathrm{~cm}$ or greater in women left atrial diameter below these value defined normal LA size (Lang MR, et al 2005).

Based on the suggestion of (Devereux RB,: et al. 1986), the following equation was applied to determine the LV mass in gram, where the mass can be determined from short-axis dimension using a simple geometric cubic formula without measuring the major axis of the LV.

$$
\text { LV mass }=0.8 \times 1.04\left[(\text { LVID }+ \text { PWT }+ \text { IVST })^{3}-\text { LVID }^{3}\right]+0.6
$$

Where LVID is the LV internal dimension, PWT is the posterior wall thickness, and IVST is the interventricular thickness. The LV mass was obtained by dividing the LV mass by the body surface area.

The LV mass index was obtained by dividing the LV mass by the body surface area.

Other Various risk factor: BP measurement in the right arm, cigarette smoking to be quantified based on daily consumption and duration of smoking. Blood biochemistry analysis was made; FBS, Cholesterol, LDL, HDL, TG, CBC

Statistical analysis: Was performed by using SPSS software (Version 12) difference among groups was analyzed by (t-test) and $\mathrm{P}$ value 0.05 was considerable significant.

\section{Result}

Table 1. shows characteristics and variation of study group.
The mean age was $69 . \pm 18$ years in the stroke group and 66 \pm 17 years in control group. There was no difference in mean age between the two groups. The number of hypertensive is significantly increased among CVA group (P 0.007). There is no significant difference in sex distribution and diabetes among groups. All smokers were male without significant difference among groups.

Table 1. Study Population Characteristics and Variations.

\begin{tabular}{lll|l}
\hline Table 1 & $\begin{array}{l}\text { Stroke group } \\
(\mathbf{n = 2 6 )}\end{array}$ & $\begin{array}{l}\text { Control group } \\
(\mathbf{n = ( 2 6 )}\end{array}$ & P value \\
\hline Age mean \pm SD & $69 \pm 18$ & $66 \pm 17 \pm$ & $\mathrm{NS}$ \\
Diabetes & $8(30 \%)$ & $4(15 \%)$ & $\mathrm{NS}$ \\
Hypertension & $16(61.5 \%)$ & $7(27 \%)$ & 0.007 \\
Smoking & $9(34.6 \%)$ & $11(42.3 \%)$ & $\mathrm{NS}$ \\
\hline
\end{tabular}

Table 2: shows Echo parameters in the stroke and control group.

The LA dimension of the stroke group $(3.82 \pm 0.50 \mathrm{~cm})$ was significantly greater than that of the control group (3.56 $\pm 0.44 \mathrm{~cm} ; \mathrm{P}<0.0001)$.

The LVEF of stroke group was not significantly different from that of the control group. Peak E and A velocity of mitral inflow were similar between the stroke and control group.

LV mass, LV mass/height, and LV mass index were significantly greater in the stroke group than in the control group $(174 \pm 46 \mathrm{~g}$ vs. $150 \pm 31 \mathrm{~g}, \mathrm{p}<0.0001107 \pm 29$ vs $94 \pm 19, \mathrm{p}=0.001106 \pm 23$ vs $95 \pm 16, \mathrm{p}=0.001$, respectively). The E/A ratio and DT were not significantly different between the two groups.

Table 2. Echocardiographic Parameters in the Stroke and Control Groups.

\begin{tabular}{llll}
\hline ECHO-parameter & Stroke group & Control group & P value \\
\hline LA Diameter $(\mathrm{cm})$ & $3.82 \pm 0.50$ & $3.56 \pm 0.44$ & $<0.0001$ \\
LVEF(\%) & $64 \pm 6.2$ & $63.5 \pm 5.4$ & $\mathrm{NS}$ \\
Mitral peak E(cm/s) & $73.3 \pm 19.2$ & $70.3 \pm 16.9$ & $\mathrm{NS}$ \\
Mitral peak A(cm/s) & $86.9 \pm 20.8$ & $84 \pm 19$ & $\mathrm{NS}$ \\
E/A ratio & $0.90 \pm 0.29$ & $0.89 \pm 0.27$ & $\mathrm{NS}$ \\
DT $(\mathrm{ms})$ & $241 \pm 50$ & $228 \pm 45$ & $\mathrm{NS}$ \\
LV mass $(\mathrm{g})$ & $174 \pm 46$ & $150 \pm 31$ & $<0.0001$ \\
LV mass / height $(\mathrm{g} / \mathrm{m})$ & $107 \pm 29$ & $94 \pm 19$ & 0.001 \\
LVMI $\left(\mathrm{g} / \mathrm{m}^{2}\right)$ & $106 \pm 23$ & $95 \pm 16$ & 0.001 \\
\hline
\end{tabular}

LV, left ventricle LVMI, left ventricular mass index LA, left atrium DT, deceleration time

Table 3: shows Echocardiographic parameters depending on the presence or absence of stroke.

To estimate the effects of hypertension on the LA dimension, the subjects were further divided into two subgroups according to the presence or absence of previous history of hypertension. For both the stroke and control group, the LA dimension did not show significant difference between subjects who did and did not have a history of hypertension $(3.91 \pm 0.5$. vs $3.73 \pm 0.47$, p $=0.1833 .64 \pm 0.45$ vs $3.55 \pm 0.46, \mathrm{p}=0.051)$. 
Table 3. Echocardiographic Parameters depending on the presence or absence of stroke.

\begin{tabular}{lllllll}
\hline & Stroke group & & \multicolumn{3}{c}{ Control group } \\
\hline Parameter & Hypertensive $(\mathbf{n}=\mathbf{1 6})$ & Normotensive $(\mathbf{n}=\mathbf{1 0})$ & P value & Hypertensive $(\mathbf{n}=\mathbf{7})$ & Normotensive $(\mathbf{n}=\mathbf{1 9})$ & P value \\
\hline LA diameter & $3.91 \pm 0.5$ & $3.73 \pm 0.47$ & 0.183 & $3.64 \pm 0.45$ & $3.55 \pm 0.46$ & 0.051 \\
LV mass $(\mathrm{g})$ & $176 \pm 45$ & $170 \pm 46$ & 0.57 & $156 \pm 30$ & $146 \pm 33$ & 0.003 \\
LV mass $/$ height $(\mathrm{g} / \mathrm{m})$ & $111 \pm 29$ & $104 \pm 33$ & 0.362 & $100 \pm 19$ & $94 \pm 19$ & 0.001 \\
LVMI $\left(\mathrm{g} / \mathrm{m}^{2}\right)$ & $111 \pm 28$ & $102 \pm 30$ & 0.234 & $99 \pm 19$ & $94 \pm 18$ & 0.003 \\
\hline
\end{tabular}

LV, left ventricle; LVMI, left ventricular mass index LA, left atrium.

Table 4: shows Echocardiographic parameters depending in the presence or absence of hypertension.

In absence of history of hypertension, LA dimension of the stroke group was greater than that of the control group (3.73 \pm 0.47 vs $3.55 \pm 0.46$, respectively $p=0.026)$, the LV mass for the stroke group was greater than that for the controls $(170 \pm 46 \mathrm{~g}$ vs $146 \pm 33 \mathrm{~g}$, respectively; $\mathrm{p}=0.008)$; However, LV mass/height and the LV mass index of the stroke group were not greater than these of the controls.

In case with history of hypertension, LA dimension of the stroke group was greater than that of the control group (3.91 \pm 0.05 vs $3.64 \pm 0.45$, respectively $p=0.002)$, the LV mass, LV mass/height, and LV mass index of the stroke group were greater than those of the controls $(176 \pm 45 \mathrm{~g}$ vs. $156 \pm 30 \mathrm{~g}$, $\mathrm{p}=0.009 ; 111 \pm 19$ vs $100 \pm 19, \mathrm{p}=0.023 ; 111 \pm 28$ vs. $99 \pm 19$, $\mathrm{P}=0.017$ )

Table 4. Echocardiographic Parameters depending on the presence or absence of Hypertension.

\begin{tabular}{|c|c|c|c|c|c|c|}
\hline \multicolumn{7}{|c|}{ Hypertensive Normotensive } \\
\hline Parameter & Stroke $(n=16)$ & Control $(n=7)$ & P value & Stroke $(n=10)$ & Control $(n=19)$ & P value \\
\hline LA diameter & $3.91 \pm 0.5$ & $3.64 \pm 0.45$ & 0.002 & $3.73 \pm 0.47$ & $3.55 \pm 0.46$ & 0.026 \\
\hline LV mass (g) & $176 \pm 45$ & $156 \pm 30$ & 0.009 & $170 \pm 46$ & $146 \pm 33$ & 0.008 \\
\hline LV mass/height (g/m) & $111 \pm 29$ & $100 \pm 19$ & 0.023 & $104 \pm 33$ & $94 \pm 19$ & 0.66 \\
\hline $\operatorname{LVMI}\left(\mathrm{g} / \mathrm{m}^{2}\right)$ & $111 \pm 28$ & $99 \pm 19$ & 0.017 & $102 \pm 30$ & $94 \pm 18$ & 0.111 \\
\hline
\end{tabular}

LV, left ventricle LVMI, left ventricular mass index LA, left atrium.

\section{Discussion}

The present case control study showed that an increase in LA dimension by itself has clear relationship with stroke in subjects presenting with preserved LVSF and normal sinus rhythm; thus neither an age older than 65 years nor reduction in LVSF is required for the relationship between LA dimension and stroke.

The Framingham heart study (Benjamin EJ, et al. 1995) had a prospective design and was able to determine the relationship between the LA dimension and stroke. The Cardiovascular health study (Tell GS, et al. 1993; Barnes $\mathrm{ME}$, et al 2004)), which also reported a relationship between the LA dimension and stroke, was performed only in subjects older than 65 year. Recently (Bong SK, et al 2009) reported that the LA dimension is related to the stroke in subjects with preserved LVSF and sinus rhythm.

The mechanisms underlying the relationship between stroke and an enlarged LA are not completely understood. The most plausible hypothesis is that LA dilation promotes stasis of blood, which in turn results in a propensity for thrombus formation and increased risk of embolism. The thrombogenicity of a dilated left atrium has been supported by the results of several trans esophageal echocardiographic studies showing an association between an enlarged left atrium and spontaneous echocardiographic contrast (Tell GS, te al 1993).

Alternatively, the LA enlargement may be a surrogate marker representing an adaptive response to some variously caused endothelial dysfunction that affects the systemic vascular bed. Endothelial dysfunction results from the abnormal regulation of and response to many different cytokines and paracrine hormones, including nitric oxide, angiotensin II and its receptor, Plasminogen inhibitor I, thrombomodulin, and endothelin (Links YT, et al. 2007)

Left atrial enlargement has frequently been associated with more severe cases of congestive heart failure, myocardial infarction, and systemic hypertension, and with chronic atrial fibrillation. Nevertheless, it is noteworthy that the relationship between stroke and LA dimension was also observed in the present study, in which the subject had preserved LVSF and normal sinus rhythm without evidence of CVD. It may be that LA enlargement promotes the development of asymptomatic interim atrial fibrillation, and this dysrhythmia cause contractile dysfunction and stasis, which further increases risk of thromboembolism.

Left atrial enlargement and left ventricular hypertrophy are manifestation of cardiac target organ damage in patients with established hypertension. In an analysis of the Framingham Heart Study cohort, Bikkina M, et al 1994, reported that increased LV mass predicts stroke. Even after adjustment for LV mass index and systemic hypertension, the LA dimension still maintained its relationship with stroke in the present study.

\section{Conclusion}

Echocardiographic left atrial enlargement has prognostic value in identifying a subset of person at increased risk for 
stroke.

Detection and treatment of hypertension prevent or reverse left ventricular hypertrophy and atrial enlargement should be an important preventive measure.

\section{References}

[1] Abhayaratna WP, Seward JB, Appleton CP, et al. Left atrial size: physiologic determinants and clinical applications. J Am coll Cardiol. 2006; 47: 2357-2363.

[2] Barnes ME, Miyasaka Y, Seward JB, et al. Left atrial volume in the prediction of first ischemic stroke in an elderly cohort without atrial fibrillation. Mayo Clin Proc. 2004; 7910081014 .

[3] Basnight MA, Gonzales MS, Keshenovich SC, Appleton CP. Pulmonary venous flow velocity: relation to hemodynamics, mitral flow velocity and left atrial volume, and ejection fraction. J Am Soc Echocardiogr. 1991; 4: 547-558.

[4] Benjamin EJ, D A gostino RB, Belanger AJ, Wolf PA, Levy D. Left atrial size and the risk of stroke and death. Circulation. 1995; 92: 835-841.

[5] Bikkina M, Levy D, Evans JC, et al. Left ventricular mass and risk of stroke in an elderly cohort. JAMA 1994; 272: 33-36.

[6] 6evereux RB, Alonso DR, Lutas EM, et al. Echocardiographic assessment of left ventricular hypertrophy: comparison to necropsy findings. Am J Cardio. 1986; 57: 450-458.

[7] Douglas PS. The left atrium: a biomarker of chronic diastolic dysfunction and cardiovascular disease risk. J Am Coll Cardiol. 2003; 42: 1206-1207.

[8] Kim SB, Lee HJ, Jang HS, Bae BS, Kang HJ, lee BR, Jong $\mathrm{BC}$, Relationship between left atrial size and stroke in patients with sinus rhythm and preserved systolic function. Korean J Intern Med. 2009 Mar 24(1): 24-32.

[9] Lang RM, Bierig M, Devereux RB, et al Recommendation for chamber quantification are part from the American society of Echocardiography and standard committee and chamber quantification writing group, developed in conjunction with European Association of Echocardiography A branch of European society of Cardiology Jam soc Echocardiography 2005; 18: 1440-63.

[10] Lee RJ, Bartzokis T, Yeoh TK, Grogin HR, Choi D, Schnittger I. Enhanced detection of intra cardiac sources of cerebral emboli by trans esophageal echocardiograph. Stroke. 1991; 22: 734-739.

[11] Links Yamashita T, Sekiguchi A, Kato T, et al. Angiotensin type 1 receptor blockade prevents endocardial dysfunction of rapidly paced atria in rats. J Renin Angiotensin Aldosterone Syst. 2007; 8: 127-132.

[12] Miller JT, O Rourke RA, Crawford MH. Left atrial enlargement: an early sign of hypertensive heart disease Am Heart J. 1988; 116: 1048-1051.

[13] Nishimura RA, Tajik AJ. Evaluation of diastolic filling of left ventricle in health and disease: Doppler echocardiography is the clinicians Rosetta Stone. J Am Coll Cardiol. 1997; 30: 818 .

[14] Oh JK, Appleton CP,Hatle LK, Nishimura RA, Seward JB, Tajik AJ. The noninvasive assessment of left ventricular diastolic function with two-dimensional and Doppler echocardiography. J Am Soc Echocardioger.1997; 10: 246270 .

[15] Tsang SM, Barnes ME, Gersh BJ, Bailey KR, Seward JB. Left atrial volume as a morphophysiologic expression of left ventricular diastolic dysfunction and relation to cardiovascular risk burden. Am J Cardiol.2002; 90: 1284-1289.

[16] Tell GS, Fried L, Hermanson B, Manolio TA, Newman AB, Borhani NO. Recruitment of adults 65 years and older as participants in the Cardiovascular Health Study. Ann Epidemiol. 1993; 3: 358-366.

[17] Weyman AE, principles and practice of echocardiography. Lee and Febiger Publ. 1994; 606.

[18] Wilson PWF, D Agostino rb, Levy D, Belanger AM, Silbershatz H, Kannel WB, prediction of coronary heart disease using risk factor categories. Circulation. 1998: 97: $1837-1847$. 